\title{
CORONARY ARTERY ANEURYSM WITH OCCLUSION DUE TO A CALCIFIED THROMBUS
}

BY

\author{
NORMAN ASHTON AND MARY MUNRO
}

From the Central Pathology Laboratory, Middle East Land Forces, and the British Military Hospital, Cyprus

Received February 12, 1948

\section{CASE RePort}

Clinical History. In January 1947 a Cypriot officer, 23 years of age, fell unconscious whilst playing football, and was dead when examined by a doctor five minutes later. His unit medical officer had known him for five years and stated that, apart from appendicectomy in 1945 and bacillary dysentery and folliculitis of the face and neck in 1946, he had been well and had led a normal active life. He had had further trouble with his folliculitis for two weeks before his death and was attending daily for local treatment, but he did not complain in any way about his general health and played football regularly.

Post-mortem examination. A well encapsulated loculated hydatid cyst, $4 \mathrm{~cm}$. in diameter, was situated in the upper part of the right lobe of the liver and the peritoneum covering the cyst was thickened. Apart from the findings in the cardiovascular system the other viscera were normal.

The left coronary artery, $5 \mathrm{~mm}$. from the aortic orifice, contained a pear shaped mass, $1.8 \mathrm{~cm}$. long and varying in width from 0.3 to $1.0 \mathrm{~cm}$., which was calcified peripherally to form a cyst-like structure and centrally it consisted of organizing laminated blood clot. In this region the artery was dilated and the walls thinned forming a cavity or recess in which the cyst lay with its broader distal end impacted in the narrow lumen beyond. There was very little atheroma and the vessel walls showed a few scattered plaques only (Fig. 1).

The right coronary artery, about $1 \mathrm{~cm}$. from its origin, was also dilated locally and there was a small indentation or pocket in the wall where marked atheromatous changes had developed. The vessel was patent. The myocardium showed softening in the posterior part of the wall of the left ventricle but there was no evidence of old infarction. All the valves were normal. Unfortunately the speci-

$\mathbf{Q}$ men was accidentally destroyed before histological preparations could be made. There can be no doubt that sudden impaction of the calcified cyst had caused occlusion of the artery and led to death.

\section{Discussion}

It was at first thought that the calcified cyst might be related to the hydatid cyst of the liver, but a

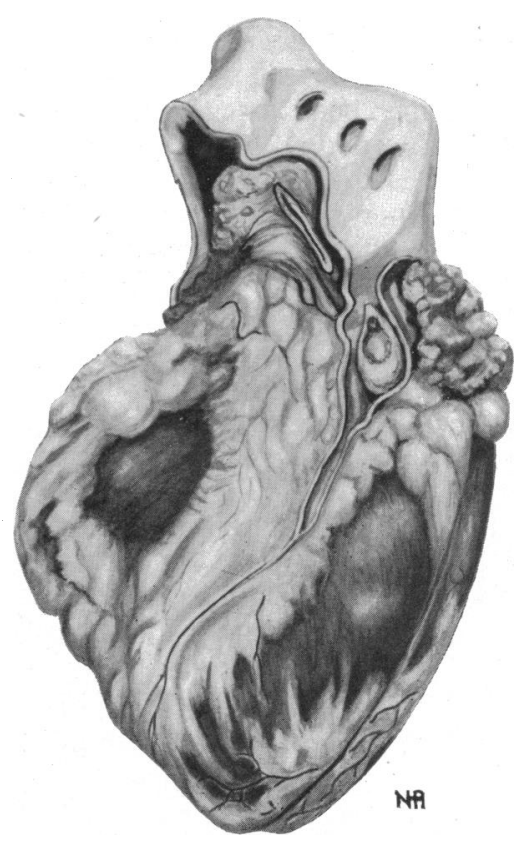

FIG. 1.-Diagrammatic drawing of the anterior aspect of the heart. The pulmonary artery has been removed and the aorta pulled forward to demonstrate the whole length of the left coronary artery. Within $5 \mathrm{~mm}$. of the ostium there is an aneurysmal dilatation containing a pear-shaped calcified cystic mass. 
hydatid seedling would be unlikely to lodge in a large artery; it would be swept on into the smaller capillaries supplying the myocardium. A hydatid cyst of the myocardium is rare; a hydatid cyst within a coronary vessel would seem to be an impossibility. The germinal epithelium would be found within the cyst but it contained old blood clot only. Furthermore the condition of the right coronary artery made it clear that the case was one of bilateral aneurysm. Within the left aneurysm a thrombus must have developed and subsequently became partially calcified. Monoher (1938) reported a case in which an oval coronary aneurysm arising $3 \mathrm{~mm}$. beyond the aortic opening contained laminated clot.

Aneurysms of the coronary vessels are well known rarities but we have been unable to discover any other reported instance of sudden death following coronary artery occlusion by a foreign body lying within the lumen of the vessel. The first case of coronary aneurysm was reported by Bougon in 1812, and Packard and Wechsler (1929) collected 29 reported cases and added one of their own. From an analysis of these cases they concluded that the condition is three times as common in men as in women and that in the vast majority of cases the aneurysm is single and is usually situated on the left coronary artery within $2.5 \mathrm{~cm}$. of its ostium. Sudden death is common and practically always due to rupture of the aneurysm; gradual heart failure is the cause of death in the others. They found no typical symptoms referable to the aneurysm itself, indeed in many cases no complaints of illness were known at the time of their sudden death. In a consideration of the atiology they classified the cases into a mycotic-embolic group, occurring in association with acute or subacute endocarditis of the aortic valve and into an arteriosclerotic group where a marked coronary sclerosis was present. Aneurysms due to periarteritis nodosa were carefully excluded from the review. Syphilitic mesaortitis was responsible for three cases in the series. In the second group they believe that the situation of the aneurysms, immediately beyond the orifice, suggests that arteriosclerosis probably precedes the aneurysmal dilatation; but when one considers the frequency of coronary sclerosis and the rarity of aneurysms, such an ætiology, even with associated hypertension, is difficult to accept. Harris (1937) in reporting a case of circoid aneurysm of the right coronary artery advanced a deficiency in the elastic lamella as a probable cause of the dilatation. More recently Rigdon and Vandergriff (1943), reviewing reported cases, consider the possible ætiology in young persons may be a solitary arteriosclerosis of the coronary vessels, though they favour congenital anomalies as more probable. They instance the work of Forbus (1930) who studied the aneurysms that develop at the point of bifurcation of the medium sized cerebral arteries, and demonstrated defects in the vessel walls; he found similar deficiencies in coronary vessels. Rigdon and Vandergriff conclude that coronary aneurysms develop in association with defects in the muscular coats located at either the point of bifurcation or at a point where a branch leaves the parent vessel.

In our case there was no evidence of syphilis, mycotic-emboli, or periarteritis nodosa, and it was clear from the advanced calcification of the thrombus that it was older than the early atheroma and it is considered that the latter was rather a resultant than a causative factor. In the absence of histological proof the exact atiology must remain a matter for speculation but we are of the opinion that the bilateral aneurysms were probably due to congenital defects in the vessel walls.

\section{SUMmaRY}

A case of bilateral coronary aneurysm in a man aged 23 is reported. Sudden death was due to impaction of a calcified thrombus in the left coronary aneurysm. The ætiology is discussed, and reasons are given for regarding it as congenital.

We wish to thank Major General F. Harris, Director of Medical Services, Middle East Land Forces, for permission to publish this case and Brigadier $C$. G. Parsons, Consultant in Medicine, for his help and advice.

\section{REFERENCES}

Bougon, (1812). Biblioth. méd., 37, 183. Forbus, W. D. (1930). Johns Hopk. Hosp. Bull., 47, 239.

Harris, P. N. (1937). Amer. J. Path., 13, 89.

Monoher, K. O. (1938). Arch. Pathol., 26, 1131.

Packard, M., and Weschler, H. F. (1929). Arch. intern. med., 43, 1 .

Rigdon, R. H., and Vandergriff, H. (1943). Amer. J. Surg., 41, 407. 